ARTICLE

DOI: $10.1038 / s 41467-018-03598-x$

\title{
Experimentally quantifying anion polarizability at the air/water interface
}

\author{
Yujin Tong ${ }^{1}$, Igor Ying Zhang ${ }^{1,2} \&$ R. Kramer Campen ${ }^{1}$
}

The adsorption of large, polarizable anions from aqueous solution on the air/water interface controls important atmospheric chemistry and is thought to resemble anion adsorption at hydrophobic interfaces generally. While the favourability of adsorption of such ions is clear, quantifying adsorption thermodynamics has proven challenging because it requires accurate description of the structure of the anion and its solvation shell at the interface. In principle anion polarizability offers a structural window, but to the best of our knowledge there has so far been no experimental technique that allowed its characterization with interfacial specificity. Here, we meet this challenge using interface-specific vibrational spectroscopy of $\mathrm{Cl}-\mathrm{O}$ vibrations of the $\mathrm{ClO}_{4}^{-}$anion at the air/water interface and report that the interface breaks the symmetry of the anion, the anisotropy of $\mathrm{ClO}_{4}^{-1}$ s polarizability tensor is more than two times larger than in bulk water and concentration dependent, and concentration-dependent polarizability changes are consistent with correlated changes in surface tension.

\footnotetext{
${ }^{1}$ Fritz Haber Institute of the Max Planck Society, 14195 Berlin, Germany. ${ }^{2}$ Department of Chemistry, Fudan University, 200433 Shanghai, China. Correspondence and requests for materials should be addressed to Y.T. (email: tong@fhi-berlin.mpg.de)
} 
T he adsorption of anions on hydrophobic interfaces controls important chemistry on aerosol surfaces and determines the stability of proteins, colloids and foams in a wide variety of environmental, physiological and engineered settings. Anion adsorption on the air/water interface, the paradigmatic hydrophobic surface, has been particularly well studied ${ }^{1-11}$. Perhaps the simplest question one can ask of this system is the following: Is anion adsorption at the air/water interface thermodynamically favourable?

In principle measurements of surface tension of the air/water interface as a function of bulk ionic strength provide such insight. Decades of such measurements have confirmed that surface tensions of aqueous salt solutions increase with increasing ionic strength, those of acids decrease, and that the magnitude of the effect depends strongly on anion and only weakly on cation ${ }^{1,12-16}$. Historically the first observation was rationalized by Wagner, Onsager and Samaras (WOS) in their extension of the Debye-Hückel theory of bulk aqueous electrolytes to interfaces. Within this description anions are excluded from the air/water interface because exposure to a low dielectric phase leads to an enormous, unfavourable electrostatic self-energy ${ }^{17,18}$. While qualitatively consistent with surface tension measurements of salt solutions, this approach does not explain surface tension trends for acids or specific ion effects.

Motivated by reaction rates of gaseous species with solvated anions in atmospheric aerosols that were unexpectedly fast ${ }^{19}$, subsequent work-simulation using classical polarizable force fields, various surface sensitive spectroscopies ${ }^{2,11,20,21}$, dielectric continuum theory ${ }^{22-25}$ and properly parameterized fixed charge models ${ }^{26}$ - has shown this Debye-Hückel inspired view to be incorrect. Large polarizable anions exist in higher concentrations at the air/water interface than in the adjoining bulk liquid. While the qualitative picture is clear, understanding of quantitative trends in anion adsorption, e.g. why does $\mathrm{I}^{-}$adsorb more strongly than $\mathrm{Cl}^{-}$, and gaining atomically resolved insight into the driving force of anion adsorption has proven challenging.

In WOS theory ions are nonpolarizable point charges. As illustrated by Levin for an idealized anion, lack of polarizability induces a large, unfavourable, electrostatic self-energy in adsorbing anions: for a finite sized, ideal, polarizable anion essentially all charge density shifts towards the aqueous phase as the ion approaches the interface ${ }^{23}$. While it is thus clear ions must be polarizable to adsorb at the air/water interface, the contribution of ion polarization (i.e. the product of applied field and polarizability $p=\alpha \cdot E)$ to the free energy of adsorption $\left(\Delta G_{\text {ads }}\right)$ is not obvious. Initial studies describing ion adsorption in classical simulations concluded that explicit description of ion polarizability was critical and that the relative surface propensity of different ions was proportional to their polarizability and radius (i.e. large, soft, polarizable anions more favourably adsorb) ${ }^{4,9}$. However subsequent simulation studies have found that anion adsorption occurs in properly parameterized classical models without explicit description of polarizability, that relative anion polarization does not correlate with experimental and simulated trends in ion adsorption propensity, and that simulated interface active anions may be similarly polarized in bulk water and at the interface $e^{21,26-30}$.

Recent theoretical work in continuum models, and classical and ab-initio simulation has clarified that $\Delta G_{\text {ads }}$ of anion adsorption is dominated by a balance between large favourable cavitation and unfavourable desolvation energies: moving an anion from bulk to the air/water interface reduces the energetic cost to forming a cavity in water but also reduces the favourable interaction of the anion with, at least part of, its solvation shell ${ }^{31-}$ 34. The contribution of ion polarization to $\Delta G_{\mathrm{ads}}$, the change in system free energy when adding a dipole to the interface, is relatively small. While accurately describing anion polarization thus appears to be relatively unimportant in decomposing anion $\Delta G_{\text {ads }}$, Pollard and Beck ${ }^{34}$ have highlighted that quantitative insight into $\Delta G_{\text {ads }}$ requires accurate description of the structure of the anion and its first solvation shell both in bulk and at the interface. Gaining such insight is a formidable theoretical challenge because the local electrical fields are as high as $1 \mathrm{~V} / \AA$. .

Much theoretical progress could be made if there were experimental observables of the structure of the anion and its first solvation shell both in bulk and at the interface. This experimental challenge is particularly formidable because the structure of the anion and its solvation shell are correlated and both might be expected to change on moving from bulk to the interface. From the monoatomic anion's perspective one might imagine structure changing because the ion electron density reflects the underlying asymmetry of electron density of the solvent, while for multiatomic anions interface induced changes in bond lengths and angles are additionally possible. From water's perspective clearly moving to the interface must result in a change in structure of the solvation shell but the details of this change must be a function of changes in anion structure. Much prior work has shown that anion polarizability is exceptionally sensitive to both anion structure and that of its local solvation shell ${ }^{35,36}$. Thus while the contribution of ion polarization to $\Delta G_{\text {ads }}$ may be small, an experimental observable of anion polarizability in bulk and at the interface should offer an important constraint on anion and solvation shell structure.

The adsorption of the perchlorate anion at the air/water interface is known to be favourable ${ }^{9}$. In this work we experimentally quantify, using interface-specific vibrational spectroscopy, the increase in polarizability anisotropy of perchlorate on moving from bulk water to the interface and show that anisotropy increases with increasing perchlorate bulk concentration. Using a simple computational model we quantitatively relate the experimentally observed increase in polarizability anisotropy with increasing bulk concentration to increases in interfacial field (consistent with prior measurements of concentration-dependent surface potential $\left.{ }^{37}\right), \mathrm{ClO}_{4}^{-}$dipole, and relative bond length of one $\mathrm{Cl}-\mathrm{O}$ bond with respect to the other three with increasing concentrations of bulk $\mathrm{HClO}_{4}$. Quantitative theoretical insights into the driving force of anion adsorption at the air/water interface, and specific ion effects more generally, require accurate calculation of both the structure of ions and their first solvation shell at aqueous interfaces. Experimental measurements of anion polarizability offer a window into such structure unavailable by other means.

\section{Results}

Optically probing the polarizability anisotropy of perchlorate. The Raman depolarization ratio $(\rho)$ is a useful means of probing anion polarizability in bulk $\mathrm{H}_{2} \mathrm{O}$. Given an isotropic distribution of ions in liquid water and a molecular coordinate system $(a, b, c)$ in which $a$ (or $b)$ is taken to be perpendicular to the net deformation of a normal mode and $c$ parallel, one writes ${ }^{38}$

$$
\rho=\frac{I_{\perp}}{I_{\|}}=\frac{3}{4+5\left(\frac{1+2 R}{R-1}\right)^{2}}
$$

where $I_{\perp}$ and $I_{\|}$are the intensity of inelastic scattered light measured perpendicular and parallel to the plane polarized incident field and $R=\frac{\partial \alpha_{a a}^{(1)} / \partial Q}{\partial \alpha_{c c}^{(1)} / \partial Q}$. That is the Raman response of a particular mode can be quantitatively related to the change in the symmetry of the molecules polarizability tensor as the molecule is deformed in the mode's characteristic manner. Given this definition of $\rho$ is it perhaps unsurprising that several studies have 

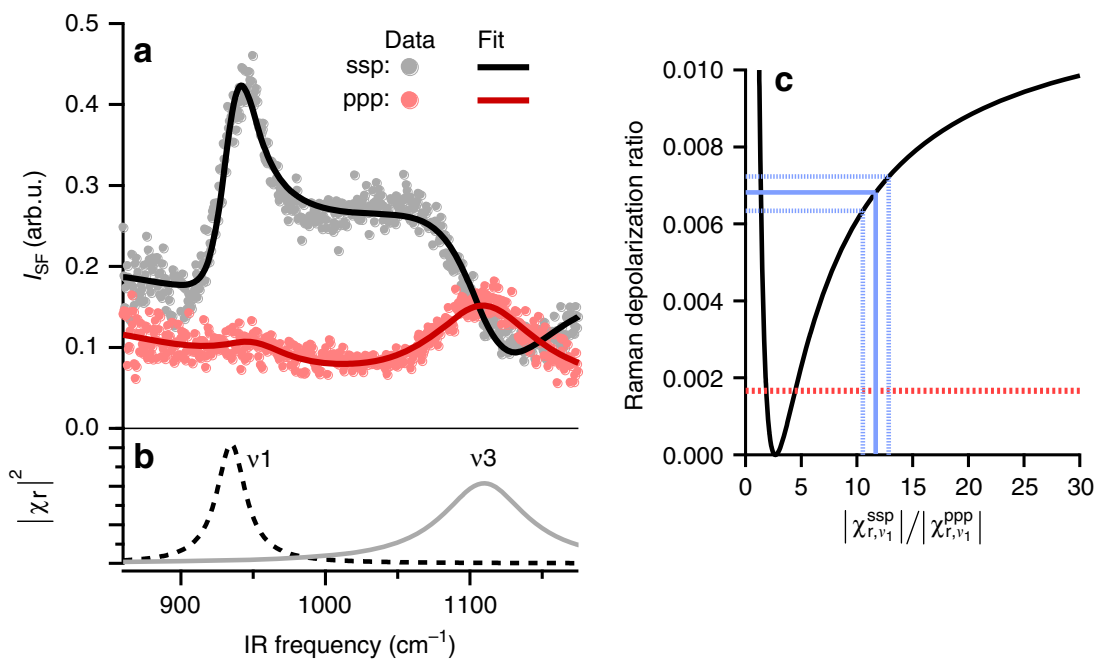

Fig. 1 The spectral response of interfacial $\mathrm{ClO}_{4}^{-}$. a SFG spectrum of $0.6 \mathrm{M} \mathrm{HClO}_{4}$ solution at the air/water interface measured under ssp (black traces) and ppp (red traces) polarization combinations. Circular symbols are the experimental observations; solid traces are the fit to Eq. (3). b Two components obtained from spectral fit, assigned to $\nu_{1}$ (A1 symmetry) and $\nu_{3}$ (F2 symmetry) vibrational modes of perchlorate respectively. c Calculated Raman depolarization ratio (black solid line) as a function of the measured VSF peak amplitude ratio $\left(\chi_{\mathbf{r}, \nu_{1}}^{\mathrm{ssp}} / \chi_{\mathrm{r}, \nu_{1}}^{\mathrm{ppp}}\right)$; the blue solid line is the result of fitting the data shown in a, blue dashed lines are the estimated uncertainty in the amplitude ratio and the resulting Raman depolarization ratio and the red dashed line indicates the bulk value reported by prior authors [43]. The uncertainty in the Raman depolarization ratio is dominated by uncertainty in the fit of the observed spectra, see Supplementary Note 1 for details of calculation

shown that the ability to calculate the Raman response of modes that are strongly coupled to the environment is a sensitive test of the accuracy of polarizability models employed ${ }^{39,40}$. Because spontaneous Raman is not interface specific it is generally not possible to extract the $\rho$ of interfacial anions. Clearly if we could, however, this observable could provide the sort of experimental constraint we seek.

Vibrationally resonant Sum Frequency (VSF) spectroscopy is a nonlinear optical, laser-based technique in which pulsed infrared (IR) and visible lasers are spatially and temporally overlapped at an interface and the output at the sum of the frequencies of the two incident beams monitored. The emitted VSF field is interface specific by its symmetry selection rules and a spectroscopy because as one tunes the frequency of one of the incident fields (in this case the IR) in resonance with an optically accessible transition the intensity of the emitted sum frequency field $\left(I_{\mathrm{sf}}\right)$ increases by several orders of magnitude. Much prior work has shown that the intensity of the measured sum frequency response at a frequency $\omega$ is proportional to the change in polarizability $\left(\alpha_{a b}\right)$ and dipole $\left(\mu_{c}\right)$ with motion along the normal mode of frequency $\omega^{41}$ :

$$
\sqrt{I_{\mathrm{sf}}} \propto \chi_{i j k}^{(2)} \propto \beta_{a b c}^{(2)} \propto-\frac{1}{2 \epsilon_{0} \omega} \frac{\partial \alpha_{a b}^{(1)}}{\partial Q} \frac{\partial \mu_{c}}{\partial Q}
$$

in which $\chi_{i j k}^{(2)}$ is the macroscopic nonlinear susceptibility in the lab coordinate system $(i j k), \beta_{a b c}^{(2)}$ the molecular hyperpolarizability and both are third rank tensors. Because by varying experimental conditions, i.e. beam incident angles and field polarizations, one can selectively probe different components of $\beta^{(2)}$, a correctly chosen ratio of intensities allows the direct measurement of $R=\frac{\partial \alpha_{a a}^{(1)} / \partial Q}{\partial \alpha_{c c}^{(1)} / \partial Q}$, and thus the possibility of extracting the Raman depolarization ratio of anions with interfacial specificity. That is, by comparing measurements of $\rho$ for an anion in solution and at the air/water interface experimental estimates of anion polarizability anisotropy at aqueous interfaces (and the change in anion polarizability anisotropy on moving from bulk liquid water to the aqueous interface) are possible.
VSF spectra of interfacial $\mathrm{ClO}_{4}^{-}$. Figure la shows the VSF spectra from the air/ $0.6 \mathrm{M} \mathrm{HClO}_{4}$ solution interface measured under the ssp ( $s$-polarized SF, $s$-polarized visible, and $p$-polarized IR) (black circles) and ppp (red squares) polarization combinations. There are two resonances apparent in this frequency range. Fitting both spectra simultaneously with the Lorentzian lineshape model described in the Methods section results in resonances centred at 935 and $1110 \mathrm{~cm}^{-1}$ (Fig. 1b dotted lines, see Supplementary Note 1 for details of the data analysis).

Because both spectral features are absent in pure water, and are spectrally separated from resonances of water or likely impurities, they can be straightforwardly assigned by reference to Raman and IR measurements of bulk aqueous perchloric acid and perchlorate salt solutions ${ }^{42-44}$. In brief, the $\mathrm{ClO}_{4}^{-}$anion has four normal modes apparent in calculation: the $v_{1}$ at 930, the $v_{2}$ at 450 , the $v_{3}$ at 1100 and the $v_{4}$ at $620 \mathrm{~cm}^{-1}$. All four are Raman active at all concentrations in bulk aqueous solution but the $v_{1}$ and $v_{2}$ are only apparent in IR absorption spectra at bulk concentrations greater than $\approx 11 \mathrm{M}$. This observation is a straightforward consequence of anion symmetry: at bulk concentrations below $11 \mathrm{M}$ the $\mathrm{ClO}_{4}^{-}$ anion has $\mathrm{T}_{d}$ symmetry (under which condition $v_{1}$ and $v_{2}$ are IR inactive) and at sufficiently high concentrations this symmetry is broken: either by ion pairing or, in the case of $\mathrm{HClO}_{4}$, by the presence of molecular acid. If we assign the resonance apparent in Fig. $1 \mathrm{~b}$ at $935 \mathrm{~cm}^{-1}$ to the $v_{1}$ mode and that apparent at $1110 \mathrm{~cm}^{-1}$ to the $v_{3}$, we are left with an apparent incongruity. As shown in Eq. (2), VSF activity requires that a mode must be both IR and Raman active. This implies the $\mathrm{ClO}_{4}^{-}$anion must lose its $\mathrm{T}_{d}$ symmetry at the air/water interface at concentrations that are at least $15 \times$ lower than those at which $\mathrm{T}_{d}$ symmetry is lifted in bulk.

Understanding why $\mathrm{T}_{\boldsymbol{d}}$ symmetry is lifted in interfacial $\mathrm{ClO}_{4}^{-}$. We imagine three possible mechanisms for the loss of $\mathrm{T}_{d}$ symmetry: consistent with recent work on other strong acids, molecular $\mathrm{HClO}_{4}$ may exist at the air/water interface at concentrations dramatically lower than in bulk ${ }^{45}, \mathrm{ClO}_{4}^{-}$may no longer have tetrahedral symmetry due to ion pairing, or it may not have tetrahedral symmetry due to, more general, solvation anisotropy 

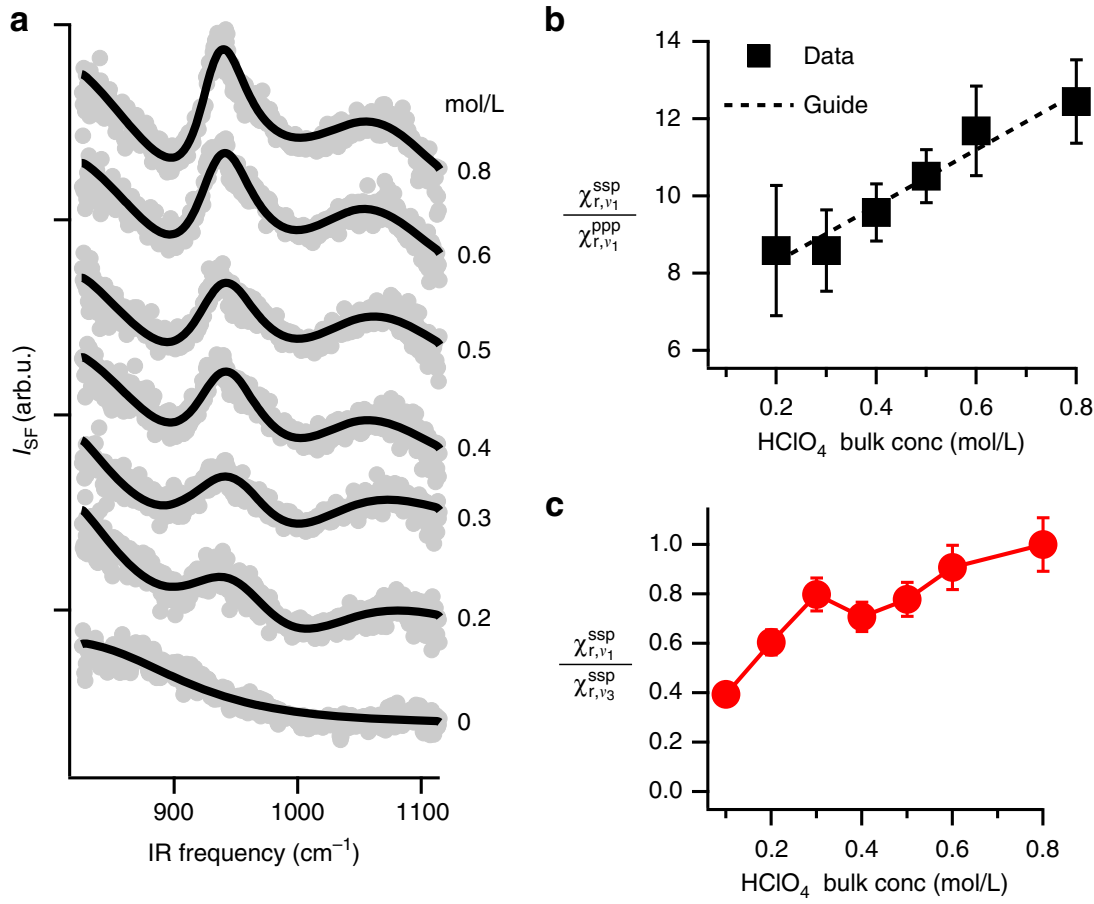

Fig. 2 The concentration-dependent spectral response of interfacial $\mathrm{ClO}_{4}^{-}$. a VSF spectra as a function of $\mathrm{ClO}_{4}^{-}$concentration below $1 \mathrm{M}$ for the ssp polarization combinations. Grey dots are data and solid black lines the corresponding fits. $\mathbf{b} \chi_{\mathbf{r}, \nu_{1}}^{\mathrm{spp}} / \chi_{\mathbf{r}, \nu_{1}}^{\mathrm{ppp}}$ for the data in a and the SI illustrating the linearity of this ratio with respect to bulk $\mathrm{HClO}_{4}$ concentration (dotted line is a guide to the eye). The uncertainty at each point is extracted from the fits to the data as described in Supplementary Note 1. c $\chi_{\mathbf{r}, \nu_{1}}^{\text {sp }} / \chi_{\mathbf{r}, \nu_{3}}^{\text {sp }}$ extracted from the data in a and the SI. This ratio varies by $\approx 50 \%$ with change in bulk concentration of $\mathrm{HClO}_{4}$. As we show in Supplementary Note 3 and discuss in the text, changes of this magnitude imply a, concentration dependent, change in interfacial $\mathrm{ClO}_{4}^{- \text {'s }}$ orientation of less than $5^{\circ}$ : the orientation of interfacial $\mathrm{ClO}_{4}^{-}$is concentration independent

at the interface. We tested the first possibility by collecting spectra from $0.6 \mathrm{M}$ solutions of $\mathrm{NaClO}_{4}$. For this a similarly intense $v_{1}$ feature is observed, suggesting the likely cause of $\mathrm{T}_{d}$ symmetry lifting is not molecular acid (see Supplementary Note 2 for data).

To evaluate the possibility of $\mathrm{T}_{d}$ symmetry lifting due to ion pairing, it is necessary to understand how ion pairing might be expected to influence the $v_{1}$ and $v_{3}$ spectral response. In bulk solutions of perchlorate salts at concentrations above $1 \mathrm{M}$ the centre frequency of perchlorate's $v_{3}$ mode has been observed to continuously shift as a function of concentration ${ }^{46}$. This concentration-dependent spectral evolution has been assigned to the formation of weak, solvent-separated ion pairs. As mentioned above, in this concentration range the $v_{1}$ mode is infrared inactive. At still higher concentrations in bulk water, $>11$ $\mathrm{M}$, perchlorate's degenerate modes, i.e. $v_{2}, v_{3}$ and $v_{4}$, have been observed to split due to contact ion pair formation, where the degree of splitting is a function of the extent to which symmetry is broken $^{46,47}$.

As is discussed in detail below (see Fig. 2 for data) at bulk concentrations lower than $1 \mathrm{M} \mathrm{HClO}_{4}$ the $v_{1}$ mode is clearly VSF (and thus IR) active, the $v_{3}$ spectral response is quantitatively reproduced with a single centre frequency and line width: splitting or frequency shift of the $v_{3}$ resonance is not required to describe our data. We therefore conclude that neither contact nor solvent-separated ion pair formation explains the lifting of $\mathrm{T}_{d}$ symmetry. Given that VSF spectra collected at bulk concentrations below $1 \mathrm{M} \mathrm{HClO}_{4}$ are consistent with formation of neither weak, solvent-separated ion pairs nor contact ion pairs, we conclude that the lifting of $\mathrm{T}_{d}$ symmetry for interfacial $\mathrm{ClO}_{4}^{-}$(and thus the IR and VSF activity of the $v_{1}$ mode) must be the result of the intrinsic anisotropy of the solvation environment at the air/ water interface: solvation anisotropy must induce sufficient structural deformation in the $\mathrm{ClO}_{4}^{-}$anion to lift the bulk $\mathrm{T}_{d}$ symmetry and make the $v_{1}$ mode IR, and VSF, active.

While this qualitative observation of a consequence of structural deformation is important, to make clear connections to theory it would be useful to quantify this deformation, and the resulting change in the perchlorate polarizability tensor. Given the $v_{1}$ spectral amplitudes extracted from the fit of the ssp and ppp data in Fig. 1a (see Supplementary Note 1 for full details of the fit), and assuming the $\mathrm{ClO}_{4}^{-}$anion is oriented such that one $\mathrm{Cl}-\mathrm{O}$ points along the surface normal and that the anion has $\mathrm{C}_{3 v}$ symmetry, we can calculate the Raman depolarization ratio, i.e. $\rho$, for perchlorate ions at the interface (see Supplementary Note 3 for a description of the theory connecting measured $I_{\mathrm{sf}}$ to the Raman depolarization ratio). The result of this calculation is shown by the solid black line in Fig. 1c. The ratio of spectral amplitudes of the $v_{1}$ ssp and ppp shown in Fig. 1a suggests a $v_{1} \rho$ of $0.0068 \pm 0.0005$ (the uncertainty originates from both the average of three measurements and the spectral fit, see Supplementary Tables 1-3 for all results), or more than $2 x$ larger than the same quantity for $\mathrm{ClO}_{4}^{-}$in bulk.

It is worth emphasizing that the result that interfacial $\mathrm{ClO}_{4}^{-}$s Raman depolarization ratio is significantly larger than bulk is insensitive to the simplifying assumptions required in its calculation. As we show in Supplementary Note 4, assuming the $\mathrm{ClO}_{4}^{-}$is oriented with one $\mathrm{Cl}-\mathrm{O}$ bond at an increasing, nonzero angle with respect to the surface normal leads to slightly larger estimates for the depolarization ratio of $v_{1}$ while assuming the symmetry of the $\mathrm{ClO}_{4}^{-}$anion has decreased to $\mathrm{C}_{2 v}$ or $\mathrm{C}_{\infty v}$ leads to quantitatively similar results. Note also that because we measure the intensity of the emitted sum frequency light, and not the field, our measurements are equally consistent with one $\mathrm{Cl}-\mathrm{O}$ bond along the surface normal and the remaining three oxygens pointing either towards the bulk liquid or air. Prior theoretical 
studies imply, at least in the low concentration limit, that the latter configuration is favoured ${ }^{7,48,49}$.

As noted above, changes in polarizability must be correlated with changes in anion nuclear structure. As we show in detail in Supplementary Note 5, a simple computational model suggests a $\rho$ of 0.0068 is consistent with a $3 \%$ change in $\mathrm{Cl}-\mathrm{O}$ bond length (for the $\mathrm{Cl}-\mathrm{O}$ along the surface normal), and a permanent dipole moment of interfacial $\mathrm{ClO}_{4}^{-}$of 0.75 Debye (n.b. consistent with the absence of IR active $v_{1}$ and $v_{2}$ modes for the $\mathrm{ClO}_{4}^{-}$anion in vacuum or in bulk liquid water, perchlorate's dipole moment in either bulk phase is below our detection limit). In this manner our experimental observable directly constrains the anisotropy of interfacial perchlorate's polarizability tensor and places quantitative constraints on interfacial $\mathrm{ClO}_{4}^{-}$polarization at the air/water interface.

Change in spectral response of interfacial $\mathrm{ClO}_{4}^{-}$with changing bulk concentration. To gain more insight into the fate of the $\mathrm{ClO}_{4}^{-}$anion at the air/water interface, we next measured its $v_{1}$ and $v_{3}$ spectral amplitudes as a function of bulk concentration of $\mathrm{HClO}_{4}$ from 0.1 to $0.8 \mathrm{M}$ (higher concentrations lead to qualitative change in the spectral response, possibly the result of interface induced ion pairing, as shown in Supplementary Note 6). The concentration-dependent spectra collected under the ssp polarization condition are shown in Fig. 2a, the concentration-dependent ppp are plotted in Supplementary Fig. 1. Global fitting of both sets of data allows the extraction of the $\chi_{\mathrm{r}, \nu_{1}}^{\mathrm{ssp}} / \chi_{\mathrm{r}, \nu_{1}}^{\mathrm{ppp}}$ ratio as a function of bulk concentration. As shown in Fig. $2 \mathrm{~b}$ changing bulk concentrations of $\mathrm{HClO}_{4}$ from 0.1 to 0.8 $\mathrm{M}$ leads to a change of this ratio from $8.58 \pm 1.7$ to $12.4 \pm 1.1$.

Because the VSF spectral response reports on the properties of interfacial $\mathrm{ClO}_{4}^{-}$, it would clearly be useful if we could quantify the increase in interfacial concentration with increasing bulk concentration of $\mathrm{HClO}_{4}$. As we discuss in detail in Supplementary Note 7 , accurately determining the interfacial concentration of anions at the interface is extremely challenging: inferring interfacial concentration from changes in surface tension with bulk concentration requires a simplifying model whose assumptions are difficult to independently evaluate, inferring interfacial population from the VSF or SHG spectral response requires assuming a concentration-independent molecular response that our results suggest is unlikely in the case of $\mathrm{ClO}_{4}^{-}$(discussed in more detail below) and, possibly, inferring interfacial concentration in $\mathrm{x}$-ray photoemission measurements requires a reference for the inelastic mean free path of the electron that does not obviously exist ${ }^{50}$. Despite these limitations we have quantified the interfacial $\mathrm{ClO}_{4}^{-}$using surface tension and VSF measurements. The surface tension data suggest that increasing bulk concentrations from $0.1 \rightarrow 1 \mathrm{M}$ lead to an increase of the surface excess of $\mathrm{ClO}_{4}^{-}$from $0.3 \times 10^{-6} \rightarrow 1.1 \times 10^{-6} \mathrm{~mol} / \mathrm{m}^{2}$ while the VSF response suggests that over this concentration range the surface coverage increases from $0.5 \rightarrow 1.0$ monolayers.

Despite the uncertainty in interfacial $\mathrm{ClO}_{4}^{-}$concentration, reference to Fig. 1 makes clear that this change $\chi_{\mathrm{r}, \nu_{1}}^{\mathrm{ssp}} / \chi_{\mathrm{r}, \nu_{1}}^{\mathrm{ppp}}$ ratio from $8 \rightarrow 13$ implies an increase in the Raman depolarization ratio of $v_{1}$ of $0.0053 \pm 0.0012$ to $0.0071 \pm 0.0004$ (all depolarization ratios with their uncertainties are tabulated in Supplementary Table 3) over the same concentration range. Evidently, with increasing interfacial population, $\mathrm{ClO}_{4}^{-}$polarizability grows increasingly anisotropic. Using the same simple computational model discussed above, increasing the depolarization ratio from 0.005 to 0.007 is consistent with an increase in interfacial field from 139 to $171(\mathrm{meV})$, an elongation in the $\mathrm{Cl}-\mathrm{O}$ bond along the surface normal of $2.6-3.3 \%$ and a change in $\mathrm{ClO}_{4}^{-}$dipole moment from 0.6 to 0.76 Debye.
The relationship between $\chi_{\mathrm{r}, \nu_{1}}^{\mathrm{ssp}} / \chi_{\mathrm{r}, \nu_{1}}^{\mathrm{ppp}}$ and Raman depolarization ratio shown in Fig. 1 assumes that the $\mathrm{ClO}_{4}^{-}$is orientated such that one $\mathrm{Cl}-\mathrm{O}$ group points along the surface normal. Applying this analysis to the data shown in Fig. 2a implicitly assumes that this orientation is concentration independent. Because the $v_{1}$ and $v_{3}$ normal modes are orthogonal, we would expect any concentration-dependent change in the orientation of interfacial $\mathrm{ClO}_{4}^{-}$to result in significant change in the $\chi_{\mathrm{r}, \nu_{1}}^{\mathrm{ssp}} / \chi_{\mathrm{r}, \nu_{3}}^{\mathrm{ss}}$. As is shown in Fig. $2 \mathrm{c}$ the change we observe in this ratio as a function of bulk concentration is $<50 \%$. A change of this size is consistent with a, concentration dependent, change in the orientation of $<5^{\circ}$ (see Supplementary Note 4 for details of the calculation). We thus conclude that the orientation of interfacial $\mathrm{ClO}_{4}^{-}$is, to within the limits of our sensitivity, over $0.1-0.8 \mathrm{M}$ range in bulk concentration, concentration independent.

Our results suggest the following model for $\mathrm{ClO}_{4}^{-}$at the air/ water interface: on adsorption $\mathrm{ClO}_{4}^{-}$is polarized, i.e. it has a nonzero dipole moment, and the polarizability anisotropy changes due to a change in the bond length of the $\mathrm{Cl}-\mathrm{O}$ that points along the surface normal relative to the three other $\mathrm{Cl}-\mathrm{O}$ bonds. With increasing interfacial concentrations of $\mathrm{ClO}_{4}^{-}$the interfacial field increases, ion polarization increases (the dipole continues to grow) and the polarizability anisotropy continues to increase.

\section{Discussion}

Modern dielectric continuum descriptions largely reproduce experimentally measured changes in surface tension with increasing concentrations of both acids and salts ${ }^{24,31}$. Notably the largest disagreements between experiment and theory, at least within the model of Levin and co-workers in which ions are approximated as hard spheres with fixed, concentrationindependent radii, exist for $\mathrm{ClO}_{4}^{-}$solutions (both acids and salts). Levin and co-workers ${ }^{51,52}$ have suggested that this is likely the result of inaccuracies in the estimates of ionic radii for $\mathrm{ClO}_{4}^{-}$. Our results are consistent with an alternative scenario in which anion polarizability (and $\mathrm{ClO}_{4}^{-}$radius) is interfacial concentration dependent. While our results imply the relationship between $\mathrm{ClO}_{4}^{-}$radius and interfacial concentration is monotonic, larger multivalent ions might be expected to have a more complicated interplay between structure, interfacial concentration and interfacial potential, all of which might be observed through the window of anion polarizability.

Atomistic simulation studies, whether employing classical or ab-initio potential energy surfaces, have largely reported either potentials of mean force for ion adsorption in the limit of infinite dilution or brute force simulations at a fixed ion concentration. As alluded to above, while important and informative these studies suffer from several possible shortcomings. The lack of experimental constraints on polarizability mean that estimates of polarizability from ab-initio simulations, and the local structure that produces them, cannot be validated and that classical polarizability models (and the manner in which they relate to local structure) cannot be parameterized. To further heighten the challenge, the surface potential of pure water is both difficult to measure experimentally and the subject of significant disagreement (by more than $0.5 \mathrm{~V}$ ) in simulation treatments $^{53}$. Thus one might expect that inaccuracies in surface potential of the pure water/air interface might, plausibly, compensate for inaccuracies in the description of local structure (and thus polarizability). Experiments of the sort described in this study gives a clear path forward through these challenges. Given experimental constraints on interfacial anion polarizability of the sort described in this study, descriptions of local anion 
structure inferred from ab-initio simulation can be validated and empirical polarizability models more accurately parameterized. Given a validated description of local ion structure systematically reducing errors in the calculation of (ion concentration dependent) surface potential is now much more straightforward.

Prior workers have performed studies similar in spirit to those shown here. Miyame et al. ${ }^{6}$ characterized the $\mathrm{S}-\mathrm{O}$ stretch vibrations of $\mathrm{SO}_{4}^{2-}$, while Motschmann and co-workers ${ }^{10}$ characterized the $\mathrm{CN}$ stretch vibrations of the potassium ferricyanide ion, i.e. $\mathrm{Fe}(\mathrm{CN})_{6}^{4-}$, as a function of bulk concentration at the air/water interface. Consistent with both calculation and other experimental approaches that suggest $\mathrm{SO}_{4}^{2-}$ retains its bulk solvation shell at the air/water interface ${ }^{4,54}$, Miyame, Morita and Ouchi find interfacial $\mathrm{SO}_{4}^{2-}$ to be essentially the same as bulk. In contrast Motschmann and co-workers found that $\mathrm{CN}$ modes that were IR inactive in bulk solution were apparent in the VSF spectrum, i.e. the interface induces a change in ferricyanide symmetry as it does for perchlorate. However, presumably because of the more structurally complicated anion, they were unable to quantify the resulting change in the polarizability tensor.

As is clear from Eq. (2), if a molecules hyperpolarizability and orientation are concentration independent one can extract a measurement of anion interfacial density as a function of bulk concentration by plotting the square root of the measured SF signal vs. bulk concentration. In a series of studies employing electronically resonant second harmonic measurements of a variety of anions at air/water interface, Saykally and co-workers, 5 have treated anion hyperpolarizability (and orientation) as concentration independent, fit adsorption isotherms to measurements of SHG signal as a function of bulk concentration, and calculated anion adsorption energies. Our results suggest that this type of data needs to be revisited. Because deformation of the perchlorate leads to an increase in transition dipole and polarizability, it is clear that given a VSF spectra collected under the ssp polarization condition, using this approach would overestimate adsorption energies (i.e. the molecular response of the perchlorate anion would increase with increasing concentration).

In summary, in the current study we have employed VSF spectroscopy and a simple computational model to study the behaviour of $\mathrm{ClO}_{4}^{-}$at the air/ $/ \mathrm{HClO}_{4}$ solution interface. Consistent with much prior work our observations clearly demonstrate that $\mathrm{ClO}_{4}^{-}$is a surface active anion. We significantly extend these prior efforts by demonstrating that the presence of the interface induces deformation of the anion that causes a bulk forbidden mode to be VSF active due to change in anion symmetry, creates a nonzero dipole moment and leads to a change in the measured polarizability anisotropy. Our results suggest that increasing density of $\mathrm{ClO}_{4}^{-}$at the interface leads to an increasing interfacial field that increases $\mathrm{ClO}_{4}^{-}$polarization (i.e. increases $\mathrm{ClO}_{4}^{-}$dipole moment), increases $\mathrm{ClO}_{4}^{-}$structural deformation and makes the polarizability of $\mathrm{ClO}_{4}^{-}$increasingly anisotropic ${ }^{4,5,9}$. Extension of the approach we describe here should allow the possibility of directly quantifying the elements of $\mathrm{ClO}_{4}^{-}$polarizability tensor, rather than just their ratio, and enable a window onto local interfacial anion structure (particularly ion/ion correlation) and the possibility of reliably experimentally quantifying the thermodynamic importance of anion polarization.

The close connection we describe here between the dipole moment, structure and polarizability of interfacial anions with increasing interfacial field has not, to our knowledge, been previously considered but should be a quite general feature of anion, particularly polyvalent anion, adsorption at hydrophobic interfaces. As such its quantitative reproduction is a prerequisite for simulation approaches that attempt to offer microscopic insight into this phenomena.

\section{Methods}

Solution preparation. $\mathrm{HClO}_{4}$ (Suprapur, 70\%, Merck) and $\mathrm{NaClO}_{4}(>99.99 \%$, Sigma-Aldrich) were used as received. Solutions with the indicated concentrations were prepared by diluting the high concentration of $\mathrm{HClO}_{4}$ and $\mathrm{NaClO}_{4}$ in ultrapure $\mathrm{H}_{2} \mathrm{O}(18.3 \mathrm{M} \Omega \mathrm{cm}$; Milli-Q, Millipore). All solutions are prepared freshly before each measurement to limit degradation or contamination. VSF measurements in the $\mathrm{C}-\mathrm{H}$ stretching and $\mathrm{C}=\mathrm{O}$ region were employed to judge the quality of the solutions.

Surface tension measurement. The concentration-dependent surface tensions were measured with a surface tensiometer (K12/K14 KRÜSS $\mathrm{GmbH})$ at room temperature $\left(25 \pm 1^{\circ} \mathrm{C}\right)$. A Du Noüy ring method with a chemically inert Pt ring was utilized. The sample volume was $50 \mathrm{~mL}$. Each measured surface tension is an average of 20 separate measurements, collected by the Pt ring in and out of the interface without tearing the lamella.

VSF measurement and spectral modelling. The VSF spectrometer employed for the current measurement, and in particular its power at long infrared wavelengths, has been described in detail in our previous studies ${ }^{55,56}$. In the interest of brevity only a brief description that pertinent to this measurement will be given here. The IR beam was generated from a commercial optical parametric amplifier (HE-TOPAS, Light Conversion) with a difference frequency generation (DFG) module. The full width half maximum (FWHM) of the beam at frequency region between 600 and $1200 \mathrm{~cm}^{-1}$ is typically $300 \mathrm{~cm}^{-1}$ with GaSe used as the DFG crystal. To probe the interfacial Cl-O stretch modes the centre frequency of the beam was tuned to $\approx 1000 \mathrm{~cm}^{-1}$. A narrow-band visible (VIS) pulse was produced from a home-made spectral shaper ${ }^{55}$. The beam is centred at $800 \mathrm{~nm}$ with a bandwidth of $15 \mathrm{~cm}^{-1}$. The energy per pulse of the IR and VIS at the sample surface was 5.8 and $15.4 \mu \mathrm{J}$ respectively. polarizations and energies of the incident fields at the interface were controlled using $\lambda / 2$ plate, polarizer, $\lambda / 2$ plate combinations. The two beams propagate in a coplanar fashion and focused on the samples using lenses with focal lengths of 10 and $25 \mathrm{~cm}$ and incident angles of $39.5 \pm 0.5^{\circ}$ and $65 \pm 0.5^{\circ}$ for the IR and VIS. All measurements were conducted in ambient conditions at room temperature and under the ssp ( $s$-polarized SF, $s$-polarized visible, and $p$-polarized IR where $p$ indicates polarization in the plane of incidence and $s$ polarization orthogonal) and ppp polarization condition. Non-resonant signals from a gold thin film were used to correct for the frequency dependent IR intensity. The acquisition time for spectra of the gold reference and samples were 30 and $300 \mathrm{~s}$, respectively.

To quantify the observed VSF spectral response, we adopted a Lorentzian lineshape model described and justified in much previous work by us and others ${ }^{55-59}$

$$
I_{\mathrm{sf}}\left(\omega_{\mathrm{sf}}\right) \propto\left|\chi_{\mathrm{eff}}^{(2)}\right|^{2} \propto|| \chi_{\mathrm{nr}}\left|\mathrm{e}^{i \epsilon}+\sum_{n} \frac{\chi_{\mathrm{r}, n}}{\omega_{\mathrm{ir}}-\omega_{n}+i \Gamma_{n}}\right|^{2}
$$

where $I_{\mathrm{sf}}\left(\omega_{\mathrm{sf}}\right)$ is the normalized VSF intensity, $\chi_{\text {eff }}^{(2)}$ is the effective second-order susceptibility, which depends on the experimental geometry, molecular hyperpolarizability and orientation. $\left|\chi_{\mathrm{nr}}\right|$ and $\epsilon$ are the non-resonant amplitude and phase and $\chi_{\mathrm{r}, n}, \omega_{n}$ and $\Gamma_{n}$ are the complex amplitude, centre frequency and line width of the $n$th resonance.

To actually analyse the data we fit the measured VSF spectrum using the Levenberg-Marquardt algorithm as implemented in the commercial visualization and analysis programme Igor Pro (Wavemetrics). Fitting spectra collected at each bulk concentration and polarization with this lineshape model results in an underdetermined minimization problem. Because bulk studies suggest that the centre frequencies and spectral shape of $\mathrm{ClO}_{4}^{-}$solution are concentration independent, we addressed these data by assuming that all spectra collected under a bulk concentration of $\mathrm{HClO}_{4}$ could be described with two resonances, each with a concentration independent line width, centre frequency and phase, and a nonresonant amplitude and phase that are also concentration independent. We accounted for the libration tail (only important in the ssp spectra) by assuming the libration has the centre frequency and line width from our previous study ${ }^{56}$. Details of the analysis, and all the parameters resulting from the fit, are given in Supplementary Note 1 .

Recent work has shown that, at sufficiently charged interfaces, it is possible that the interfacial $\chi^{(2)}$ signal of specifies also present in the adjoining bulk aqueous phase, may be distorted by a $\chi^{(3)}$ response from oscillators within the electrical double layer. Such mixing is unlikely to effect the $v_{1} \mathrm{Cl}-\mathrm{O}$ spectral response (this mode is symmetry inactive away from the interface and thus has no spectral response within the double layer) and unlikely to effect the VSF spectra of either the $v_{1}$ or $v_{3}$ at the relatively low interfacial charge densities but high electrolyte concentration we explore here ${ }^{60-62}$.

Data availability. All data is available from the corresponding author on request. 
Received: 26 October 2017 Accepted: 19 February 2018

Published online: 03 April 2018

\section{References}

1. Weissenborn, P. K. \& Pugh, R. J. Surface tension of aqueous solutions of electrolytes: relationship with ion hydration, oxygen solubility, and bubble coalescence. J. Colloid Interface Sci. 184, 550-563 (1996).

2. Ghosal, S. et al. Electron spectroscopy of aqueous solution interfaces reveals surface enhancement of halides. Science 307, 563-566 (2005).

3. Cheng, J., Vecitis, C. D., Hoffmann, M. \& Colussi, A. Experimental anion affinities for the air/water interface. J. Phys. Chem. B 110, 25598-25602 (2006).

4. Jungwirth, P. \& Tobias, D. J. Specific ion effects at the air/water interface. Chem. Rev. 106, 1259-1281 (2006).

5. Petersen, P. B. \& Saykally, R. J. On the nature of ions at the liquid water surface. Ann. Rev. Phys. Chem. 57, 333-364 (2006).

6. Miyamae, T., Morita, A. \& Ouchi, Y. First acid dissociation at an aqueous $\mathrm{H}_{2} \mathrm{SO}_{4}$ interface with sum frequency generation spectroscopy. Phys. Chem. Chem. Phys. 10, 2010-2013 (2008).

7. Baer, M. D., Kuo, I.-F. W., Bluhm, H. \& Ghosal, S. Interfacial behavior of perchlorate versus chloride ions in aqueous solutions. J. Phys. Chem. B 113, 15843-15850 (2009).

8. Hua, W., Verreault, D. \& Allen, H. C. Surface prevalence of perchlorate anions at the air/aqueous interface. J. Phys. Chem. Lett. 4, 4231-4236 (2013).

9. Tobias, D. J., Stern, A. C., Baer, M. D., Levin, Y. \& Mundy, C. J. Simulation and theory of ions at atmospherically relevant aqueous liquid-air interfaces. Ann. Rev. Phys. Chem. 64, 339-359 (2013).

10. Brandes, E., Karageorgiev, P., Viswanath, P. \& Motschmann, H. Breaking the symmetry of ions at the air-water interface. J. Phys. Chem. C 118, 26629-26633 (2014).

11. Hua, W., Verreault, D. \& Allen, H. C. Relative order of sulfuric acid, bisulfate, hydronium, and cations at the air-water interface. J. Am. Chem. Soc. 137, 13920-13926 (2015).

12. Heydweiller, A. Über physikalische eigenschaften von lösungen in ihrem zusammenhang. II. oberflächenspannung und elektrisches leitvermögen wässeriger salzlösungen. Ann. Phys. 338, 145-185 (1910).

13. Randles, J. Ionic hydration and the surface potential of aqueous electrolytes. Discuss. Faraday Soc. 24, 194-199 (1957)

14. Randles, J. E. B. \& Schiffrin, D. J. Surface tension of dilute acid solutions Trans. Faraday Soc. 62, 2403-2408 (1966).

15. Matubayasi, N., Matsuo, H., Yamamoto, K., Yamaguchi, S. \& Matuzawa, A. Thermodynamic quantities of surface formation of aqueous electrolyte solutions: I. Aqueous solutions of $\mathrm{NaCl}, \mathrm{MgCl}_{2}$, and $\mathrm{LaCl}_{3}$. J. Colloid Interface Sci. 209, 398-402 (1999).

16. Matubayasi, N. et al. Thermodynamic quantities of surface formation of aqueous electrolyte solutions: IV. Sodium halides, anion mixtures, and sea waters. J. Colloid Interface Sci. 243, 444-456 (2001).

17. Wagner, C. The surface tension of diluted electrolyte solutions. Phys. Z. 25, 474-477 (1924).

18. Onsager, L. \& Samaras, N. N. T. The surface tension of Debye-Hückel electrolytes. J. Chem. Phys. 2, 528-536 (1934).

19. Knipping, E. M. et al. Experiments and simulations of ion-enhanced interfacial chemistry on aqueous $\mathrm{NaCl}$ aerosols. Science 288, 301-306 (2000).

20. Padmanabhan, V. et al. Specific ion adsorption and short-range interactions at the air aqueous solution interface. Phys. Rev. Lett. 99, 086105 (2007).

21. Otten, D. E., Shaffer, P. R., Geissler, P. L. \& Saykally, R. J. Elucidating the mechanism of selective ion adsorption to the liquid water surface. Proc. Natl. Acad. Sci. USA 109, 701-705 (2012).

22. Boström, M., Williams, D. R. M. \& Ninham, B. W. Surface tension of electrolytes: specific ion effects explained by dispersion forces. Langmuir 17, 4475-4478 (2001).

23. Levin, Y. Polarizable ions at interfaces. Phys. Rev. Lett. 102, 147803 (2009).

24. Levin, Y., dos Santos, A. P. \& Diehl, A. Ions at the air-water interface: an end to a hundred-year-old mystery? Phys. Rev. Lett. 103, 257802 (2009).

25. Wang, R. \& Wang, Z.-G. Continuous self-energy of ions at the dielectric interface. Phys. Rev. Lett. 112, 136101 (2014)

26. Netz, R. R. \& Horinek, D. Progress in modeling of ion effects at the vapor/ water interface. Ann. Rev. Phys. Chem. 63, 401-418 (2012).

27. Caleman, C., Hub, J. S., van Maaren, P. J. \& van der Spoel, D. Atomistic simulation of ion solvation in water explains surface preference of halides. Proc. Natl. Acad. Sci. USA 108, 6838-6842 (2011).

28. Baer, M. D. \& Mundy, C. J. Toward an understanding of the specific ion effect using density functional theory. J. Phys. Chem. Lett. 2, 1088-1093 (2011)

29. Baer, M. D., Stern, A. C., Levin, Y., Tobias, D. J. \& Mundy, C. J. Electrochemical surface potential due to classical point charge models drives anion adsorption to the air-water interface. J. Phys. Chem. Lett. 3, 1565-1570 (2012).

30. Sun, L., Li, X., Tu, Y. \& Agren, H. Origin of ion selectivity at the air/water interface. Phys. Chem. Chem. Phys. 17, 4311-4318 (2015).

31. Duignan, T. T., Parsons, D. F. \& Ninham, B. W. Ion interactions with the air? water interface using a continuum solvent model. J. Phys. Chem. B 118, 8700-8710 (2014)

32. Ben-Amotz, D. Interfacial solvation thermodynamics. J. Phys. Condens. Matter 28, 414013 (2016).

33. Duignan, T. T., Baer, M. D. \& Mundy, C. J. Ions interacting in solution moving from intrinsic to collective properties. Curr. Opin. Colloid Interface Sci. 23, 58-65 (2016)

34. Pollard, T. P. \& Beck, T. L. Toward a quantitative theory of Hofmeister phenomena: from quantum effects to thermodynamics. Curr. Opin. Colloid Interface Sci. 23, 110-118 (2016).

35. Lopez, M. A. \& Montero, S. Raman intensities, vibrational eigenvectors, electro-optical parameters and force constants of $\mathrm{SO}_{4}{ }^{2-}, \mathrm{ClO}_{4}{ }^{-}, \mathrm{PO}_{4}{ }^{3}, \mathrm{CO}_{3}{ }^{2-}$ and $\mathrm{NO}_{3}{ }^{-}$anions in polycrystalline samples.J. Raman Spectrosc. 9, 386-392 (1980).

36. Ye, P. \& Shen, Y. R. Local-field effect on linear and nonlinear optical properties of adsorbed molecules. Phys. Rev. B 28, 4288-4294 (1983).

37. Marcus, Y. Specific ion effects on the surface tension and surface potential of aqueous electrolytes. Curr. Opin. Colloid Interface Sci. 23, 94-99 (2016)

38. Hirose, C., Akamatsu, N. \& Domen, K. Formulas for the analysis of surface sum-frequency generation spectrum by $\mathrm{CH}$ stretching modes of methyl and methylene groups. J. Chem. Phys. 95, 997-1004 (1992).

39. Hamm, P. 2D-Raman-THz spectroscopy: a sensitive test of polarizable water models. J. Chem. Phys. 141, 184201 (2014).

40. Ito, H., Hasegawa, T. \& Tanimura, Y. Effects of intermolecular charge transfer in liquid water on Raman spectra. J. Phys. Chem. Lett. 7, 4147-4151 (2016).

41. Lambert, A. G., Davies, P. B. \& Neivandt, D. J. Implementing the theory of sum frequency generation vibrational spectroscopy: a tutorial review. Appl. Spectrosc. Rev. 40, 103-145 (2005)

42. Ratcliffe, C. \& Irish, D. Vibrational spectral studies of solutions at elevated temperatures and pressures. VI. Raman studies of perchloric acid. Can. J. Chem. 62, 1134-1144 (1984).

43. Hyodo, S.-A. Depolarization of the $v_{1}$ Raman band of $\mathrm{ClO}_{4}{ }^{-}$in $\mathrm{LiClO}_{4} /$ ethylene carbonate-water solution.Chem. Phys. Lett. 161, 245-248 (1989).

44. Karelin, A. \& Grigorovich, Z. Vibrational spectra of perchloric acid-II. Modifications and phase transitions of solid $\mathrm{HClO}_{4}$ and $\mathrm{DClO}_{4}$. Spectrochim. Acta A 32, 851-857 (1976).

45. Baer, M. D., Tobias, D. J. \& Mundy, C. J. Investigation of interfacial and bulk dissociation of $\mathrm{HBr}, \mathrm{HCl}$, and $\mathrm{HNO}_{3}$ using density functional theorybased molecular dynamics simulations. J. Phys. Chem. C 118, 29412-29420 (2014).

46. Chen, Y., Zhang, Y.-H. \& Zhao, L.-J. ATR-FTIR spectroscopic studies on aqueous $\mathrm{LiClO}_{4}, \mathrm{NaClO}_{4}$, and $\mathrm{Mg}\left(\mathrm{ClO}_{4}\right)_{2}$ solutions. Phys. Chem. Chem. Phys. 6, 537-542 (2004)

47. Ritzhaupt, G. \& Devlin, J. P. Infrared spectra of matrix isolated alkali metal perchlorate ion pairs. J. Chem. Phys. 62, 1982-1986 (1975).

48. Ottosson, N. et al. Large variations in the propensity of aqueous oxychlorine anions for the solution/vapor interface. J. Chem. Phys. 131, 124706 (2009).

49. Hey, J. C., Smeeton, L. C., Oakley, M. T. \& Johnston, R. L. Isomers and energy landscapes of perchlorate/water clusters and a comparison to pure water and sulfate-water clusters. J. Phys. Chem. A 120, 4008-4015 (2016).

50. Olivieri, G. et al. Quantitative interpretation of molecular dynamics simulations for $\mathrm{x}$-ray photoelectron spectroscopy of aqueous solutions. $J$. Chem. Phys. 144, 154704 (2016).

51. dos Santos, A. P., Diehl, A. \& Levin, Y. Surface tensions, surface potentials, and the Hofmeister series of electrolyte solutions. Langmuir 26, 10778-10783 (2010).

52. Levin, Y. \& dos Santos, A. P. Ions at hydrophobic interfaces. J. Phys. Condens. Matter 26, 203101 (2014)

53. Kathmann, S. M., Kuo, I.-F. W., Mundy, C. J. \& Schenter, G. K. Understanding the surface potential of water. J. Phys. Chem. B 115, 4369-4377 (2011).

54. Gopalakrishnan, S., Liu, D., Allen, H. C., Kuo, M. \& Shultz, M. J. Vibrational spectroscopic studies of aqueous interfaces: salts, acids, bases, and nanodrops. Chem. Rev. 106, 1155-1175 (2006).

55. Tong, Y. et al. Optically probing $\mathrm{Al}-\mathrm{O}$ and $\mathrm{O}-\mathrm{H}$ vibrations to characterize water adsorption and surface reconstruction on $\alpha$-Alumina: an experimental and theoretical study. J. Chem. Phys. 142, 054704 (2015).

56. Tong, Y., Kampfrath, T. \& Campen, R. K. Experimentally probing the libration of interfacial water: the rotational potential of water is stiffer at the air/water interface than in bulk liquid. Phys. Chem. Chem. Phys. 18, 18424-18430 (2016). 
57. Tong, Y., Vila Verde, A. \& Campen, R. K. The free OD at the air $/ \mathrm{D}_{2} \mathrm{O}$ interface is structurally and dynamically heterogeneous. J. Phys. Chem. B 117, 11753-11764 (2013).

58. Bain, C. D., Davies, P. B., Ong, T. H., Ward, R. N. \& Brown, M. A Quantitative analysis of monolayer composition by sum-frequency vibrational spectroscopy. Langmuir 7, 1563-1566 (1991).

59. Zhuang, X., Miranda, P. B., Kim, D. \& Shen, Y. R. Mapping molecular orientation and conformation at interfaces by surface nonlinear optics. Phys. Rev. B 59, 12632-12640 (1999).

60. Gonella, G., Lütgebaucks, C., de Beer, A. G. F. \& Roke, S. Second harmonic and sum-frequency generation from aqueous interfaces is modulated by interference. J. Phys. Chem. C 120, 9165-9173 (2016).

61. Wen, Y.-C. et al. Unveiling microscopic structures of charged water interfaces by surface-specific vibrational spectroscopy. Phys. Rev. Lett. 116, 016101 (2016).

62. Ohno, P., Wang, H., Skinner, J., Paesani, F. \& Geiger, F. M. On second-order vibrational lineshapes of the air/water interface. Preprint at https://arxiv.org/ abs/1712.09086 (2017).

\section{Acknowledgements}

The authors thank Irina Shekova for helping with the surface tension measurement and Martin Wolf for useful discussions and the Max Planck Society for support of this work.

\section{Author contributions}

Y.T. and R.K.C. designed the study. Y.T. performed the measurements. Y.T. and R.K.C. analysed the data and wrote the paper. I.Y.Z. performed the electronic structure calculations. All authors edited the paper.

\section{Additional information}

Supplementary Information accompanies this paper at https://doi.org/10.1038/s41467018-03598-x.

Competing interests: The authors declare no competing interests.

Reprints and permission information is available online at http://npg.nature.com/ reprintsandpermissions/

Publisher's note: Springer Nature remains neutral with regard to jurisdictional claims in published maps and institutional affiliations.

(c) (i) Open Access This article is licensed under a Creative Commons Attribution 4.0 International License, which permits use, sharing, adaptation, distribution and reproduction in any medium or format, as long as you give appropriate credit to the original author(s) and the source, provide a link to the Creative Commons license, and indicate if changes were made. The images or other third party material in this article are included in the article's Creative Commons license, unless indicated otherwise in a credit line to the material. If material is not included in the article's Creative Commons license and your intended use is not permitted by statutory regulation or exceeds the permitted use, you will need to obtain permission directly from the copyright holder. To view a copy of this license, visit http://creativecommons.org/ licenses/by/4.0/.

(C) The Author(s) 2018 